\title{
Readers' Credits for Volume 21, Issue 1
}

Studies in Applied Linguistics \& TESOL (SALT) would like to thank the following individuals for reviewing the contributions for Volume 21, Issue 1 (2021):

\section{Contributing Reviewers}

\author{
Ahyoung Alicia Kim \\ WIDA
}

\section{Ashley Beccia}

Teachers College, Columbia University

\section{Carol Lo}

Teachers College, Columbia University

Erik Voss

Teachers College, Columbia University

Haimei Sun

Teachers College, Columbia University

\section{Jorge Beltrán}

Teachers College, Columbia University

Joshua Raclaw

West Chester University

Midori Ishida

San Jose State University
Nadja Tadic

Teachers College, Columbia University

Paige Yi

Teachers College, Columbia University

\section{Renka Ohta}

Educational Testing Service

Saerhim Oh

Pearson

\section{Sarah Creider}

Teachers College, Columbia University

\section{Sarah Sok}

University of California, Irvine

Soo Hyoung Joo

Teachers College, Columbia University

Taichi Yamashita

Iowa State University 